\title{
EDITORIAL
}

\section{LEPROSY IN POST ELIMINATION ERA}

Vikash Paudel ${ }^{1}$, Brijesh Shrestha ${ }^{1}$

${ }^{1}$ Journal Editor, Medphoneix, Journal of National Medical College, Birgunj, Nepal

Citation: Paudel V, Shrestha B. Leprosy in Post elimination era. Medphoenix. 2021;6(2):1

DOI: 10.3126/medphoenix.v6i2.42540
Available at www.jnmc.com.np ISSN:2631-1992 (Online); ISSN:2392-425X (Print)

(i) This work is licensed under a Creative Commons Attribution 4.0 International License.
Leprosy is a chronic granulomatous infectious disease caused by Mycobacterium leprae, an acid-fast bacillus, which mostly affects the skin and peripheral nerves. It is considered as a major public health problem in many countries including Nepal. ${ }^{1}$ It is often mentioned as the oldest disease known to man, the origins of which are lost in the mists of antiquity. The cardinal signs of leprosy are hypoaesthetic or anesthetic, erythematous or hypopigmented patch, peripheral nerve function impairment, and presence of acid fast bacilli in the slit skin smear or biopsy. It is considered transmitted from person to person following prolonged close proximity, but leprosy is curable with appropriate treatment. The treatment regimen of leprosy consists of multidrug therapy with Rifampicin, Clofazamine, and Dapsone. Without appropriate treatment, it can lead to deformities and blindness, and therefore early detection and treatment is crucial. ${ }^{2}$

Despite of Nepal has achieved the elimination of leprosy as a public health problem in December 2009 and declared elimination in 2010, it is still regarded as a public health problem. Nepal is one of the 22 global priority countries for the disease according to the World Health Organization (WHO). ${ }^{3}$ In 2019/2020, the registered prevalence rate (PR) was 0.69 cases per 10,000 population at the national level. This prevalence rate is lower than the recorded PR of the previous year of 0.99 . Out of 77 districts, 11 districts reported zero prevalence, 56 districts had a prevalence rate $<1$, and 10 districts had a prevalence rate of more than 1 case per 10,000 population. The number of districts reporting a prevalence rate of more than 1 case per 10,000 population decreased to 10 districts from 17 in the previous year. Lalitpur district reported the highest prevalence rate of 4.28 cases per 10,000 population among all 10 districts with $P R>1$. Other districts with prevalence more than 1 case per 10000 were Jhapa, Banke, Dhanusha, Nawalparasi West, Parsa, Kailali, Kapilbastu, Rupandehi and Achham. ${ }^{4}$

Besides, the stigma associated with leprosy is also high in the terrain region because of the lower socioeconomic and education status of the population. Recently, leprosy in Nepal was in an increasing trend, thus it is considered as a reemerging threat. ${ }^{5}$ There have been significant changes regarding the disease burden of leprosy at the national level, but in the few pocket districts of Nepal bordering with India, the prevalence rate is very higher than the national average. ${ }^{4}$

Thus, sustaining the achievement at the national level \& further reducing the disease burden in a local level is still challenging. Although, there is the high burden of leprosy in local community diseases compared to the national average, the public health program directed to leprosy is minimum and patients are unaware of the clinical features and proper management of leprosy. The stigma associated with the disease in the community hinders in proper management of the disease.

\section{REFERENCES}

1) Jain MC. Leprosy scenario in Nepal. J Nepal Med Assoc. 2008;47(172):259-63 [DOl]

2) World Health Organisation. Leprosy: Key Facts. WHO; 2019 [Full Text]

3) World Health Organization, Regional Office for South-East Asia. Global Leprosy Strategy 20162020: Accelerating towards a leprosy-free world. Operational Manual. New Delhi: WHO; 2016. [Full Text]

4) Leprosy Control and Disability Management Program Annual. 2021. [Full Text]

5) Houghton F, Winterburn M. Leprosy in Nepal: a re-emerging threat. J Public Health Policy. 2021 Mar;42(1):176-181. [DOI] 